\title{
Risk factors for oxygen desaturation on arrival in the postanesthesia care unit
}

\author{
Matthew Walker, BEng • Robert G. Farmer, PhD • \\ Ben Schelew, MD
}

Received: 25 November 2014/ Accepted: 20 March 2015/Published online: 26 March 2015

(c) Canadian Anesthesiologists' Society 2015

\section{To the Editor,}

Hypoxemia is a common complication in patients arriving in the postanesthesia care unit (PACU) following surgery. Its associated consequences have led the Canadian Anesthesiologists' Society (CAS) to recommend the use of supplemental oxygen during transport to the PACU "when clinically indicated". ${ }^{1}$ Although the CAS does not define which patients are at risk for desaturation, previous research has identified a number of risk factors, including pre-existing comorbidities, surgery type, anesthetic technique and transport while breathing room air., ${ }^{2,3}$

We conducted a quality assurance study of patient desaturation to address the risks of transport-related hypoxemia in the context of our unique hospital setting. Our hospital has operating rooms (OR) on the 10th and 11th floors, but the only PACU is on the 10th floor, necessitating elevator transport between certain ORs and the PACU. Our associated mean (SD) transport time is two to three times longer than the mean time at other institutions [3.7 (2.0) min vs 1-1.5 min, respectively]. ${ }^{2,4}$ Compared with similar previous work, this study had a larger sample size and fewer exclusions.

With hospital Research Ethics Board approval (November, 2013), we retrospectively audited records of adult patients who received a general anesthetic requiring PACU admission from September 1, 2012 to August 31, 2013 at the Victoria General Hospital in Halifax, Nova Scotia. We excluded patients who arrived in the PACU with hypothermia (temperature $<35.0^{\circ} \mathrm{C}$ ). We used data from 3,808 patients in multilevel (mixed-effects) linear, and logistic models to identify independent predictors for

M. Walker, BEng $(\bowtie) \cdot$ R. G. Farmer, PhD - B. Schelew, MD Faculty of Medicine, Dalhousie University, Halifax, NS, Canada oxygen desaturation during transport between the OR and the PACU. We considered age, sex, body mass index (BMI), preoperative $\mathrm{SpO}_{2}$, surgical duration, transport duration, use of supplemental oxygen during transport, and the use of elevator transport. We controlled for variations among surgery types as random effects.

During the study period, 2,201 (57.8\%) postoperative patients were transported to the PACU on supplemental oxygen. Among the patients transported to the PACU on room air, $71(4.4 \%)$ patients desaturated from $\geq 90 \%$ (recorded electronically on departure from the OR) to $<90 \%$ on the first saturation recorded by the PACU nurse. Only $13(0.6 \%)$ patients among those transported on supplemental oxygen desaturated to $<90 \%$.

A multilevel linear regression identified six predictors of a decline in $\mathrm{SpO}_{2}(P=0.014$ or less; Table): older age, higher BMI, lower preoperative $\mathrm{SpO}_{2}$, longer surgery, absence of supplemental oxygen during transport, and lack of elevator transport. Among these predictors, the absence of supplemental oxygen was the most influential, with the highest standardized coefficient (data not shown) and absolute t-value by a wide margin (Table). Without supplemental oxygen, model simulations predicted a mean (SD) decline in $\mathrm{SpO}_{2}$ of $2.7(0.2) \%$ between the $\mathrm{OR}$ and the PACU. With supplemental oxygen, model simulations predicted a mean (SD) gain of $0.7(0.2) \%$ relative to OR departure values. Similarly constructed multilevel logistic models predicted the mean (SD) overall risks of transport desaturation from $\geq 90 \%$ to $<90 \%$ to be $4.5(0.9) \%$ without supplemental oxygen. This modelled risk decreased to $0.3(0.1) \%$ with oxygen use for a calculated absolute risk reduction of $4.2 \%$ and a calculated NNT of 24 .

Consistent with previous research, ${ }^{2}$ the longer duration of transport to the PACU was not a risk factor here (though one smaller study identified duration of transport as a risk factor). ${ }^{5}$ 
Table Patient demographics and predictors of change in $\mathrm{SpO}_{2}$ during transport between the OR and the PACU

\begin{tabular}{|c|c|c|c|c|c|}
\hline \multicolumn{5}{|l|}{ Demographics } & \multirow{2}{*}{$\frac{(n=3,808)}{1,949(51.18)}$} \\
\hline \multicolumn{5}{|l|}{ Male, $n(\%)$} & \\
\hline \multicolumn{5}{|l|}{ Age yr, mean (SD) } & $56.0(16.3)$ \\
\hline \multicolumn{5}{|l|}{$\mathrm{BMI} \mathrm{kg} \cdot \mathrm{m}^{-2}$, mean $(\mathrm{SD})$} & $29.5(6.8)$ \\
\hline \multicolumn{5}{|c|}{ Surgery (relative \%) (A/E/G/O/P/R/S/T) } & $21 / 23 / 7 / 1 / 33 / 5 / 3 / 7$ \\
\hline \multicolumn{5}{|c|}{ Preoperative (baseline) $\mathrm{SpO}_{2}$, mean $\%$ (SD) } & $97.5(1.7)$ \\
\hline Predictors of change in $\mathrm{SpO}_{2}$ & Coefficient $(\beta)$ & SE & t-value & $P$ value & $95 \% \mathrm{CI}$ \\
\hline (Intercept) & -13.183 & 3.769 & -3.498 & $<0.001$ & $(-20.57$ to -5.80$)$ \\
\hline Age* $^{*}$ & -0.016 & 0.004 & -4.237 & $<0.001$ & $(-0.023$ to -0.008$)$ \\
\hline Male sex & 0.012 & 0.117 & 0.099 & 0.461 & $(-0.218$ to 0.241$)$ \\
\hline BMI* & -0.019 & 0.009 & -2.205 & 0.014 & $(-0.037$ to -0.002$)$ \\
\hline Preoperative (baseline) $\mathrm{SpO}_{2} *$ & 0.123 & 0.037 & 3.315 & $<0.001$ & (0.05 to 0.196$)$ \\
\hline Surgery duration* & -0.112 & 0.042 & -2.678 & 0.004 & $(-0.195$ to -0.03$)$ \\
\hline Transport duration to PACU & 0 & 0.001 & -0.010 & 0.496 & $(-0.001$ to 0.001$)$ \\
\hline Supplemental $\mathrm{O}_{2}$ during transport* & 3.398 & 0.129 & 26.348 & $<0.001$ & (3.145 to 3.651$)$ \\
\hline Elevator effect* & 0.002 & 0.001 & 3.533 & $<0.001$ & (0.001 to 0.003$)$ \\
\hline
\end{tabular}

Coefficients with negative values indicate an association with oxygen desaturation; positive values may indicate a protective effect. The larger the magnitude of the coefficient the more influential the effect

*Non-zero estimates $(P<0.05)$; BMI = body mass index; OR = operating room; PACU $=$ postanesthesia care unit; $\mathrm{SE}=$ standard error Surgery type: $\mathrm{A}=$ abdominal; $\mathrm{E}=\mathrm{ENT}$ (ear, nose, and throat); $\mathrm{G}=$ groin; $\mathrm{O}=$ oropharynx; $\mathrm{P}=$ pelvis; $\mathrm{R}=$ renal; $\mathrm{S}=$ skin + breast; $\mathrm{T}=$ thorax

Elevator effect $=$ our hospital has ORs on the tenth and 11th floors, but the only PACU is on the 10th floor. We hypothesized that anesthesiologists are more vigilant with 11th floor patients before departing the OR to protect against the putative increased risks associated with transport to the PACU requiring time in a small elevator. Because of this hypothesis we accounted for the "elevator effect" in our model

Interestingly, we found that patients having surgery on the floor closest to the PACU were at increased risk of a decline in $\mathrm{SpO}_{2}$ compared with patients from ORs on the 11th floor. We speculate that patients on the 11th floor were likely better oxygenated because anesthesiologists were more vigilant given the necessity of elevator travel to reach the PACU.

Although a very small percentage of our patients $(0.6 \%)$ desaturated to $<90 \%$ even when transported on supplemental oxygen, the overwhelming protective effect of oxygen supplementation in limiting hypoxemia leads us to conclude that it should continue to be considered after every general anesthetic, especially in higher risk patients. Future studies on the cost-effectiveness of an increased use of supplemental oxygenation could be revealing.

Disclosures This work was unfunded. None of the authors have any commercial or non-commercial affiliations that are or may be perceived to be a conflict of interest with the work, and none of the authors have any other associations, such as consultancies, that are or may be perceived to be a conflict of interest with the work.

\section{References}

1. Merchant $R$, Chartrand D, Dain $S$, et al. Guidelines to the practice of anesthesia - revised edition 2015. Can J Anesth 2015; 62: 54-79.

2. Siddiqui N, Arzola C, Teresi J, Fox G, Guerina L, Friedman Z. Predictors of desaturation in the postoperative anesthesia care unit: an observational study. J Clin Anesth 2013; 25: 612-7.

3. Moller JT, Wittrup M, Johansen SH. Hypoxemia in the postanesthesia care unit: an observer study. Anesthesiology 1990; 73: 890-5.

4. Xue FS, Huang YG, Tong SY, et al. A comparative study of early postoperative hypoxemia in infants, children, and adults undergoing elective plastic surgery. Anesth Analg 1996; 83: 709-15.

5. Blair I, Holland R, Lau W, McCarthy N, Chiah TS, Ledwidge D. Oxygen saturation during transfer from operating room to recovery after anaesthesia. Anaesth Intensive Care 1987; 15: 147-50. 